\title{
Tumores primarios del yeyuno-íleon: correlación clínico-patológica*
}

\author{
Drs. GUILLERMO BANNURA C. ${ }^{1}$, ALEJANDRO BARRERA E. ${ }^{1}$, \\ CARLOS MELO L. ${ }^{1}$, FELIPE ILLANES F. ${ }^{1}$ \\ 1 Servicio y Departamento de Cirugía, Hospital Clínico San Borja Arriarán. Campus Centro, Facultad de Medicina, \\ Universidad de Chile. \\ Santiago, Chile.
}

\begin{abstract}
\section{Jejunoileal primary tumors. Experience in 24 patients}

Background: Short bowel tumors correspond to $2 \%$ of gastrointestinal tract tumors and are the third cause of bowel obstruction. Aim: To perform a clinico-pathological correlation of jejunoileal tumors. Patients and Methods: Retrospective revision of medical records of patients operated for a primary jejunoileal tumor in a period of 17 years, excluding duodenal tumors. Results: Twenty four patients were identified, seven had gastrointestinal stromal tumors (GIST), six had a carcinoid tumor and five had lymphomas. GIST predominantly involved distal jejunum and proximal ileum, while carcinoid tumors and lymphomas tended to involve the distal ileum. The main clinical presentation of GIST was gastrointestinal bleeding. Carcinoid tumors presented mostly as bowel occlusion and lymphomas as bowel perforation. Benign lesions tended to present as intussusception. CAT scan and CAT enteroclysis allowed the preoperative diagnosis in 20 patients. Immunohistochemistry was relevant for the pathological diagnosis and radical surgery was the basis of treatment. Prognosis depended on the pathology of the tumor, the degree of malignancy and the tumor stage at the moment of diagnosis. Conclusions: Excluding duodenum, GIST and carcinoid tumors account for $65 \%$ of primary malignant tumors of jejunum and ileum. Some clues for the diagnosis can be obtained from the clinical picture of the patients.
\end{abstract}

Key words: Jejunoileal tumors, GIST, carcinoid.

\section{Resumen}

Objetivo: Establecer una correlación clínico-patológico de los tumores primarios de yeyuno e íleon (YI). Pacientes y Método: Revisión retrospectiva que incluye todos los pacientes intervenidos por un tumor primario de Y-I, con exclusión de los tumores de duodeno, en un período de 17 años. Resultados: 24 pacientes, destacando 7 tumores GIST, 6 carcinoides y 5 linfomas. Predominio de sexo masculino (20/4), distribución por edad variable según el tipo de tumor (promedio 55,5 años en los GIST, 64 años en los tumores carcinoides y 50 años en los linfomas). Es llamativo el compromiso del yeyuno y de íleon proximal de los GIST, mientras que los tumores carcinoides y el linfoma afectan de preferencia al íleon terminal. En la presentación clínica

*Recibido el 5 de diciembre de 2011 y aceptado el 12 de enero de 2012.

Correspondencia: Dr. Guillermo Bannura C. Las Limas 1622, Santiago, Chile. gbannura@vtr.net 
predomina la hemorragia digestiva en los GIST, la obstrucción intestinal incompleta en los carcinoides, la perforación en los linfomas, la obstrucción aguda en los adenocarcinomas y la intususcepción en las lesiones benignas. La tomografía computada (TC) y la enteroclisis por TC permitió el diagnóstico preoperatorio en 20 pacientes. La inmunohistoquímica (IHQ) es relevante en el diagnóstico histopatológico de certeza y la cirugía radical con R0 es la base del tratamiento de estas lesiones. El pronóstico depende del tipo histológico, el grado de malignidad y la etapa al momento del diagnóstico. Excluyendo el duodeno, el GIST y los tumores carcinoides representan más de la mitad del global y el 65\% de las neoplasias malignas primarias que afectan el segmento Y-I. Conclusión: A pesar de la rareza y de la heterogeneidad de estos tumores, es posible reconocer una correlación clínico patológica útil en el manejo quirúrgico de los tumores primarios de Y-I.

Palabras clave: Tumores primarios de yeyuno e íleon.

\section{Introducción}

Los tumores primarios del intestino delgado (ID) constituyen alrededor del $2 \%$ de todas las neoplasias del tubo digestivo, representan la tercera causa de obstrucción del ID y generalmente son diagnosticados en etapas avanzadas ${ }^{1}$. Dado el alcance de los estudios endoscópicos, el diagnóstico preoperatorio de las lesiones distales al ángulo de Treitz ha sido un desafío para el clínico, situación que ha cambiado en los últimos años con la introducción de la tomografía computada (TC) de múltiple corte y, más recientemente, la enteroclisis por $\mathrm{TC}^{2}$. La mayoría de los estudios incluyen los tumores del duodeno, que si bien forma parte del ID, tiene características propias que creemos es conveniente tratarlas por separado ${ }^{3-5}$. Los tumores primarios del yeyuno-íleon incluyen lesiones tanto benignas como malignas de origen muy diverso y se considera que la forma de presentación clínica es muy inespecífica. En general, se describe un cuadro de dolor abdominal de larga data, baja de peso, alteraciones del tránsito intestinal, hemorragia digestiva y en el extremo del espectro, obstrucción intestinal y/o perforación ${ }^{5}$. Señalar en forma global los síntomas más relevantes de estos tumores supone una forma similar de presentación clínica para tumores de diferente etiología y comportamiento biológico muy dispar. El objetivo de este trabajo es establecer si existe una correlación clínico-patológica de los tumores primarios de yeyuno-íleon y analizar los resultados en el manejo de estos tumores.

\section{Pacientes y Método}

Se trata de un estudio retrospectivo y descriptivo de todos los pacientes sintomáticos intervenidos por un tumor primario del yeyuno y del íleon desde el ángulo de Treitz hasta la válvula ileocecal (VIC) en el período octubre 1993 - marzo 2011. Se excluyen los tumores del duodeno, el compromiso por vecindad de tumores originados en otros órganos o en el contexto de una enfermedad metastásica y los hallazgos de lesiones incidentales (asintomáticas) del segmento en estudio. Se registró la distribución por edad y género, la duración de los síntomas, los exámenes que orientaron el diagnóstico, la ubicación de las lesiones, la histología y el tratamiento efectuado. Se intenta establecer una correlación entre la forma de presentación clínica, la forma macroscópica y el tipo histológico de la lesión. Se utilizó la clasificación TNM (AJCC, sexta edición) ${ }^{6}$ para los adenocarcinoma y los carcinoides y la clasificación Ann Arbor para evaluar la extensión de los linfomas ${ }^{7}$. Para los criterios histológicos de malignidad de los GIST se empleó el consenso de $2002^{8}$. La cirugía se consideró radical si el tumor se resecó en forma completa o paliativa en caso contrario. El seguimiento se efectuó mediante controles en el policlínico de la especialidad y los archivos del Registro Civil.

\section{Resultados}

En el período señalado se intervinieron 24 pacientes por un tumor primario de yeyuno e íleon, destacando el GIST como el tumor más común en esta serie, seguido del carcinoide y el linfoma, constituyendo el 75\% de los casos. La correlación entre los síntomas y el tipo macroscópico del tumor se aprecia en la Tabla 1.

La edad promedio de los tumores GIST fue 55,5 años (extremos 38-72), 5 hombres, la localización fue en el yeyuno proximal en 4 casos y en la porción yeyuno-ileal en 3. El síntoma principal fue la hemorragia digestiva baja (HDB) reiterada con anemia secundaria, que se manifestó como hemorragia oculta en 3 casos y en forma macroscópica en 2. Otros síntomas destacados fueron el dolor abdominal en 3 casos y la masa abdominal en 2 . Salvo el caso $n^{0} 3$, la baja de peso no fue un síntoma llamativo. El diagnóstico se efectuó mediante la laparotomía exploradora en 1 caso, la enteroclisis por TC en un caso y la tomografía computada (TC) abdominal en el resto. El TC demostró una masa sólida exofítica en relación a un segmento de yeyuno-íleon con refuerzo 
Tabla 1. Correlación clínico-patológica de los tumores primarios de yeyuno-íleon

\begin{tabular}{|c|c|c|c|}
\hline Tipo histológico & $\mathbf{n}$ & Cuadro clínico & Anat. Patológica (macroscopia) \\
\hline GIST s & 7 & $\begin{array}{l}\text { HDB (4), masa (2), } \\
\text { Invaginación (1) }\end{array}$ & Tumor sólido, 4 a 11 cm, exofítico \\
\hline Carcinoides & 6 & Obstrucción intestinal & $\begin{array}{l}\text { Lesión protruida tipo placa, firme, única o múltiple, de } 1 \text { a 5,5 cm, } \\
\text { gran masa indurada del mesenterio }\end{array}$ \\
\hline Linfomas & 5 & $\begin{array}{l}\text { Perforación intestinal (2), } \\
\text { HDBM (1), obstrucción (2) }\end{array}$ & $\begin{array}{l}\text { Múltiples lesiones ulceradas ( } 3,5 \text { a } 12 \text { cm). Tumor ulcerado } 13 \\
\mathrm{~cm} \text {. }\end{array}$ \\
\hline \multicolumn{4}{|l|}{ Hamartomas } \\
\hline (S. Peutz-Jeghers) & 2 & Invaginación intestinal & Lesiones protruidas múltiples de 1,5 a $8 \mathrm{~cm}$ \\
\hline Adenocarcinoma & 1 & Obstrucción intestinal & Bormmann II 4 cm, infiltra ciego y vejiga \\
\hline Leiomiosarcoma & 1 & Obstrucción intestinal & Tumor elevado, duro, 4,5 x 4,5 cm \\
\hline Pólipo inflamatorio & 1 & Invaginación & Tu polipoideo de $6 \mathrm{~cm}$ \\
\hline Lipoma & 1 & Invaginación ileocecal & Tu submucoso $3,3 \mathrm{~cm}$, blando \\
\hline
\end{tabular}

HDB: hemorragia digestiva baja; M: masiva.

periférico intenso por el contraste endovenoso. En un caso de un tumor gigante, la tomografía describió una masa retroperitoneal sólido-quística de $20 \mathrm{~cm}$, confirmándose una lesión mixta de 20 x 18 x 6,5 que pesó 1.700 gramos (Caso $n^{\circ} 3$ ). En el contexto del estudio de una hemorragia digestiva, dos pacientes fueron sometidos a múltiples exámenes endoscópicos, cintigrafías y arteriografías selectivas. En todos los casos se practicó una resección segmentaria con linfadenectomía limitada y anastomosis inmediata sin morbilidad significativa. El tamaño del tumor fluctuó entre 4 y $20 \mathrm{~cm}$, de consistencia firme y con crecimiento exofítico en 6 (Figura 1). Un paciente con un tumor elevado de $4,5 \mathrm{~cm}$ de crecimiento intraluminal fue intervenido de urgencia por una invaginación intestinal. La histopatología describe una proliferación neoplásica de células fusiformes con un recuento mitótico que fluctuó entre 0 y 10 mitosis por campo de aumento mayor y áreas de necrosis muy variables. De acuerdo a la clasificación del 2002, los tumores fueron clasificados como malignos en 3 casos, de bajo potencial maligno en

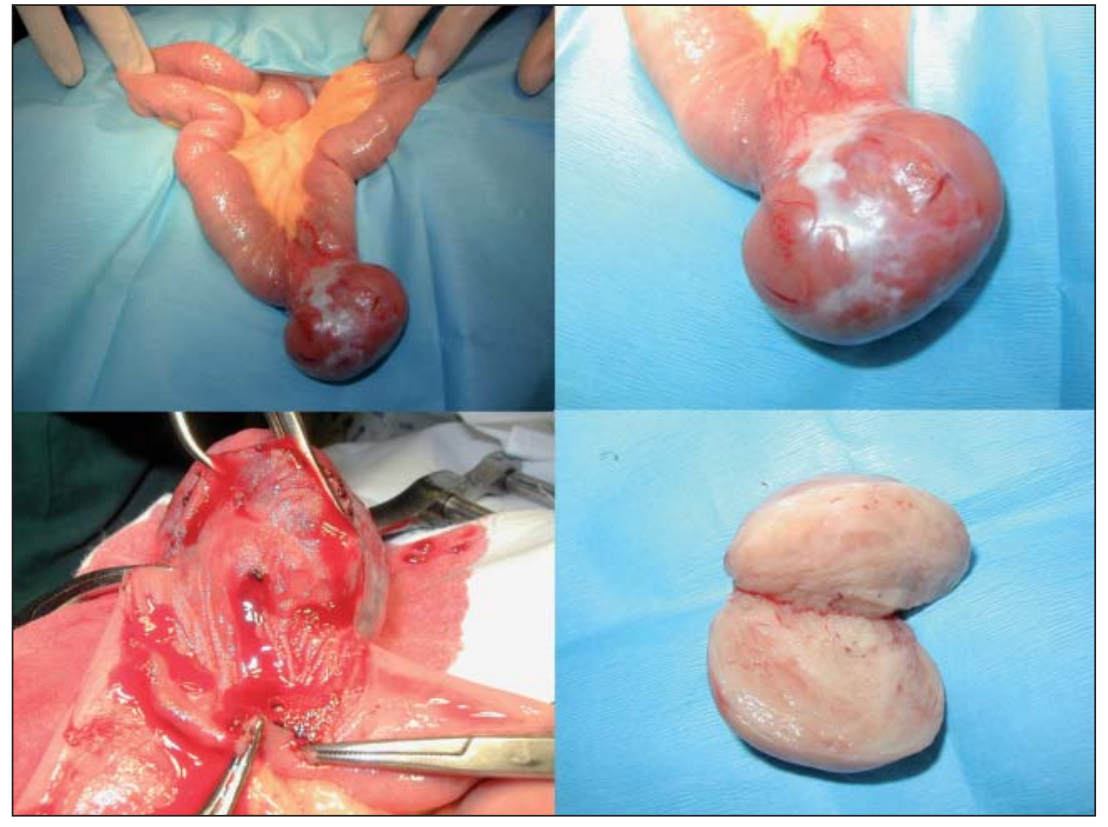

Figura 1. GIST del íleon: tumor exofítico, sólido, complicado con una HDB. 
2 y de potencial maligno intermedio en 2. Las técnicas de Inmunohistoquímica mostraron un CD 117 positivo en los 5 casos en que se efectuó, CD 34 en 3 de 4, AML (actina musculo liso) positivo focal en 3 de 4 y S 100 positivo focal en 1 caso. Algunas características de los tumores GIST y el seguimiento a largo plazo se detalla en la Tabla 2.

En el grupo de los 6 tumores carcinoides (Tabla 2) (cinco varones y una mujer) con un promedio de edad de 63,8 años (extremos 48-77), hubo 4 casos de lesiones múltiples (2 a 5 lesiones) de 1 a 5,5 cm de diámetro mayor. La forma de presentación clínica fue en todos ellos una obstrucción intestinal crónica o incompleta de larga data (8 a 40 meses), destacando el dolor, la baja de peso (12 a 30 kilos) y el compromiso del estado general (Tablas 1 y 2). Un paciente $\left(n^{\circ} 3\right)$ con una historia de 4 años de dolor cólico, baja de peso de $20 \mathrm{~kg}$ fue intervenido en un servicio de urgencia 2 meses previo a su ingreso por obstrucción intestinal, efectuándose un by pass entero-enteral, siendo resecado posteriormente en nuestro Servicio. La TC abdominal sugirió el diagnóstico de tumor intestinal en todos los casos y de un tumor carcinoide en 4. La TC mostró una masa/ engrosamiento de la pared del intestino y/o una masa en la raíz del mesenterio que se contrasta en forma intensa con marcada reacción desmoplástica y gra- dos variables de dilatación proximal del intestino. En una paciente el TC mostró múltiples imágenes focales hepáticas. La colonoscopia reveló en dos pacientes una lesión neoplásica de íleon distal y de la válvula ileocecal con biopsia positiva para carcinoide en ambos casos. Se efectuó una resección radical segmentaria con íleo-tiflectomía y anastomosis inmediata en 5 pacientes y entero-enteroanastomosis en la restante. La pieza operatoria mostró una o múltiples lesiones de consistencia firme tipo placa con una gran reacción del mesenterio adyacente, retracción del meso, angulación y dilatación proximal (Figura 2). En todos los pacientes el tumor infiltraba toda la pared intestinal con extensión al mesenterio. La IHQ realizada en 3 casos reveló una tinción intensa con cromogranina, citoqueratina AE1-AE3 moderada focal a intensa y sinaptofisina enolasa neuroespecífica intensa en las células neoplásicas. En dos pacientes (una de ellas con metástasis hepáticas) se indicó quimioterapia adyuvante con platino y etopóxido.

Hubo 5 casos de linfoma primario de intestino delgado, todos varones con un promedio de edad de 49 años (extremos 32-68), que debutan como emergencias quirúrgicas debido a una perforación intestinal (2 casos), hemorragia digestiva baja masiva (1 caso) y obstrucción intestinal (2 casos). Un

Tabla 2. Diagnóstico y seguimiento de tumores primarios de yeyuno-íleon

\begin{tabular}{|c|c|c|c|c|c|c|}
\hline Edad/Género & Clínica & Diagnóstico & Segmento & $\begin{array}{l}\text { Tipo de } \\
\text { lesión }\end{array}$ & $\begin{array}{l}\text { Follow up } \\
\text { meses }\end{array}$ & $\begin{array}{c}\text { Recidiva } \\
\text { RL/RD }\end{array}$ \\
\hline \multicolumn{7}{|l|}{ GIST } \\
\hline $65 / \mathrm{H}$ & Invaginación & Laparotomía & Yeyuno & Maligna & 4 & $\mathrm{RD}$ \\
\hline $72 / \mathrm{H}$ & Masa abdominal & $\mathrm{TC}$ & Yeyuno-íleon & Maligna & 63 & no \\
\hline $41 / \mathrm{H}$ & Masa abdominal & TC & Yeyuno & Maligna & 25 & $R L+R D$ \\
\hline $55 / \mathrm{H}$ & HDB & TC & $\begin{array}{l}\text { Yeyuno-íleon } \\
\text { Intermedia }\end{array}$ & 104 & no & \\
\hline $38 / \mathrm{H}$ & HDB & TC & Yeyuno & Intermedia & 100 & no \\
\hline $47 / \mathrm{M}$ & HDB & TC & Yeyuno & Benigna & 96 & no \\
\hline $71 / \mathrm{M}$ & HDB & Enteroclisis & Yeyuno-íleon & Benigna & 26 & no \\
\hline \multicolumn{7}{|c|}{ Tumor carcinoide } \\
\hline 71/H & Dolor, < peso $20 \mathrm{k}$ & TC & Íleon distal & Múltiple & $7^{*}$ & no \\
\hline 77/H & Dolor, < peso 7 k, melena & TC + Colonoscopia & VIC & Único & 62 & no \\
\hline $67 / \mathrm{H}$ & Dolor, < peso $20 \mathrm{k}$ & TC & Íleon distal & Múltiple & 36 & no \\
\hline $48 / \mathrm{H}$ & Dolor, < peso 12 k & TC & Íleon distal & Múltiple & 6 & no \\
\hline $56 / \mathrm{H}$ & $\begin{array}{l}\text { Dolor, < peso } 30 \mathrm{k} \text {, } \\
\text { masa abdominal }\end{array}$ & TC + Colonoscopia & Íleon distal & Único & 6 & no \\
\hline 61/M & Dolor, < peso 14 k & TC & Íleon distal* & Múltiple & 6 & no \\
\hline
\end{tabular}

HDB: hemorragia digestiva baja. VIC: válvula ileocecal. TAC: tomografía computada. RL: recidiva local. RD: recidiva a distancia. *Metástasis hepáticas (5). *Fallece por infarto del miocardio libre de enfermedad. 


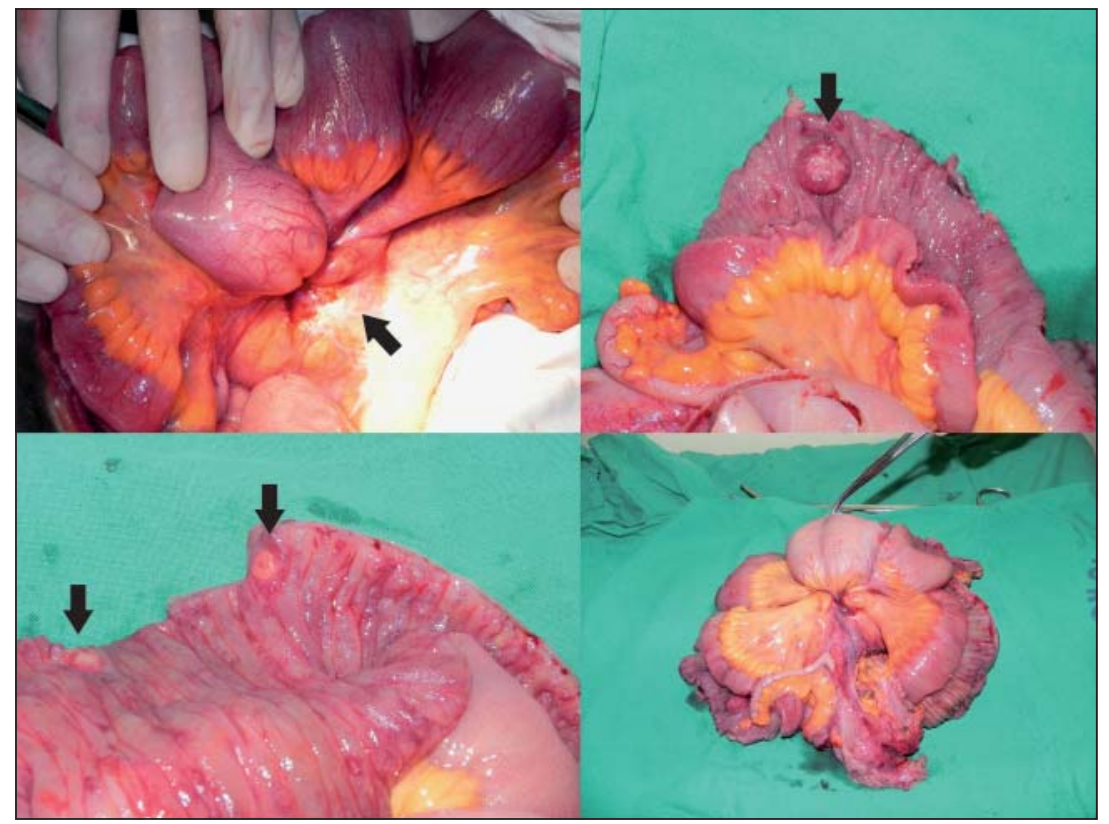

Figura 2. Carcinoide del íleon: lesiones múltiples tipo placa con gran invasión del mesenterio que angula y obstruye el lumen intestinal. paciente de 32 años con una historia de dolor, baja de peso $10 \mathrm{k}$ y marcado compromiso del estado general ingresa con una perforación intestinal y es sometido a una exploración y resección con anastomosis inmediata. La pieza demostró múltiples lesiones ulceradas y el diagnóstico fue linfoma difuso no Hodgkin multicéntrico de células grandes de alto grado (tipo mediterráneo) con compromiso focal en linfonodos. Fue sometido a 3 ciclos de quimioterapia (COPP-Bleo) seguido de radioterapia abdominal con 3.000 Rads que fue bien tolerada, recuperando 18 kilos, pero fallece a los 19 meses con enfermedad diseminada. Un paciente hipertenso y coronario de 68 años ingresa con un cuadro de obstrucción intestinal incompleta, masa palpable y baja de peso de 12 k. LA TC reveló una masa pelviana para-ileal de 15 x $6 \mathrm{~cm}$ con compromiso del fondo vesical. Se efectuó una resección de íleon y colon derecho involucrados en una masa tumoral de gran tamaño con anastomosis inmediata. El paciente es reintervenido a los 7 días por dehiscencia de sutura y fallece a los 21 días por sepsis y falla multisistémica. El estudio histopatológico demostró un linfoma difuso no Hodgkin de alto grado (células pequeñas no clivadas) de $13 \mathrm{~cm}$ de longitud del íleon distal con compromiso transmural y compromiso por vecindad de segmentos proximales de íleon y del ciego. Un paciente de 48 años ingresa en caquexia (IMC = 17), con una reactivación de una tuberculosis y es intervenido de urgencia por una perforación intestinal. Se comprueba ascitis, dos lesiones tumorales en íleon a 30 y $70 \mathrm{~cm}$ de la VIC, además de 2 lesiones tumores en ciego y colon transverso izquierdo. Se efectuó una resección de íleon más una colectomía derecha extendida con ileostomía terminal y fístula mucosa del colon. El paciente fallece a los 5 días en falla multiorgánica secundaria a la sepsis. La biopsia de la pieza quirúrgica reveló múltiples lesiones ulceradas en íleon de 4, 2 y $4 \mathrm{~cm}$, de $6 \mathrm{~cm}$ en colon transverso y de $12 \mathrm{~cm}$ en el ciego y que comprometen todo el espesor del intestino, con compromiso de 16 de 26 ganglios examinados (Figura 3). Conclusión: Linfoma intestinal de tipo T, difuso, pleomórfico, de alto grado, ulcerado. IHQ: CD3 positivo intenso, CD 30 negativo, CD 15 negativo, CD 68 negativo y CD 20 negativo en células neoplásicas y positivo focal en linfocitos de aspecto reactivo. Un paciente HIV + de 48 años ingresa a pabellón con una hemorragia digestiva baja masiva con diagnóstico endoscópico de tumor ulcerado de íleon distal sangrante a $50 \mathrm{~cm}$ de la VIC, falleciendo a las 24 horas de la intervención. Biopsia reveló una proliferación linfoide atípica de células grandes, con marcado pleomorfismo nuclear, ulcerada, compatible con linfoma difuso no Hodgkin de células grandes de alto grado. El quinto paciente consulta por dolor, vómitos y baja de peso de $18 \mathrm{k}$ en 2 meses. Enteroclisis por TC revela engrosamiento parietal del yeyuno con dilatación proximal, sugerente de linfoma, asociado a compromiso pulmonar izquierdo. Se realizó una resección parcial paliativa debido a compromiso retroperitoneal extenso, evolucionando con una fístula de yeyuno proximal de manejo difícil, falleciendo a los 3 meses por progresión de la enfermedad. La IHQ mostró CD20 y bcl-2 


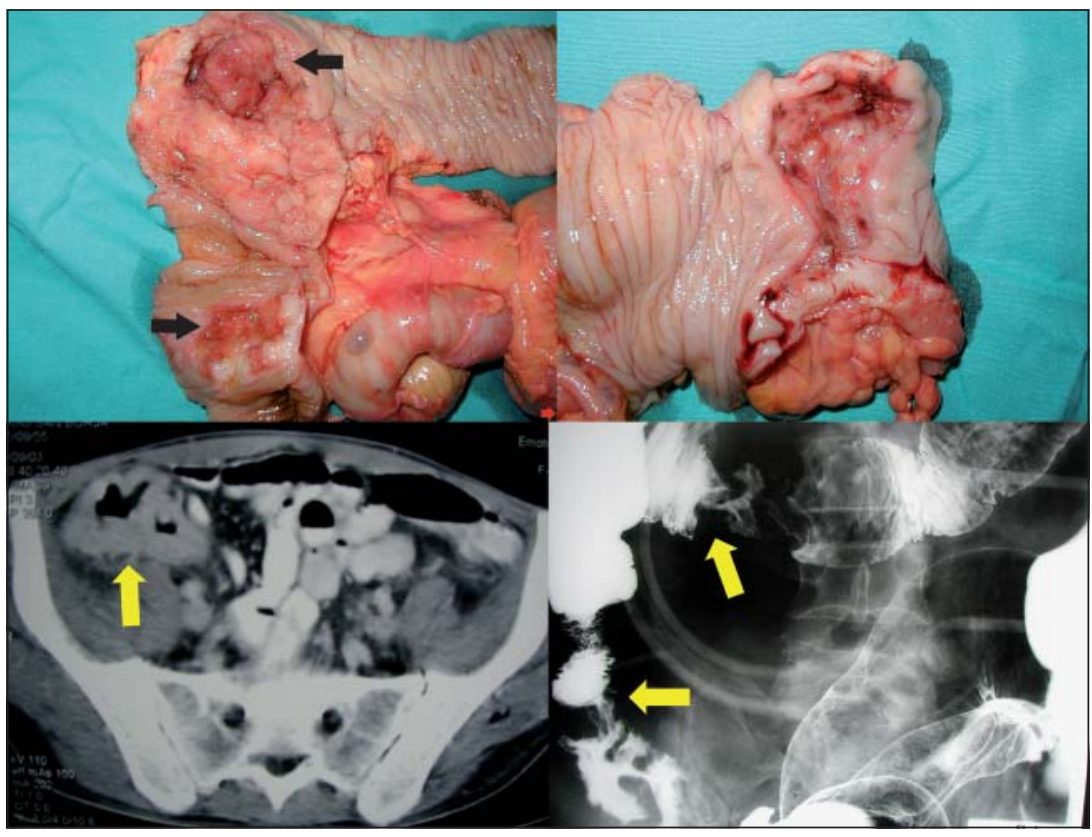

Figura 3. Linfoma del íleon, ciego y colon transverso: lesiones ulceradas e infiltrativas múltiples (TC, enema baritado y pieza operatoria).

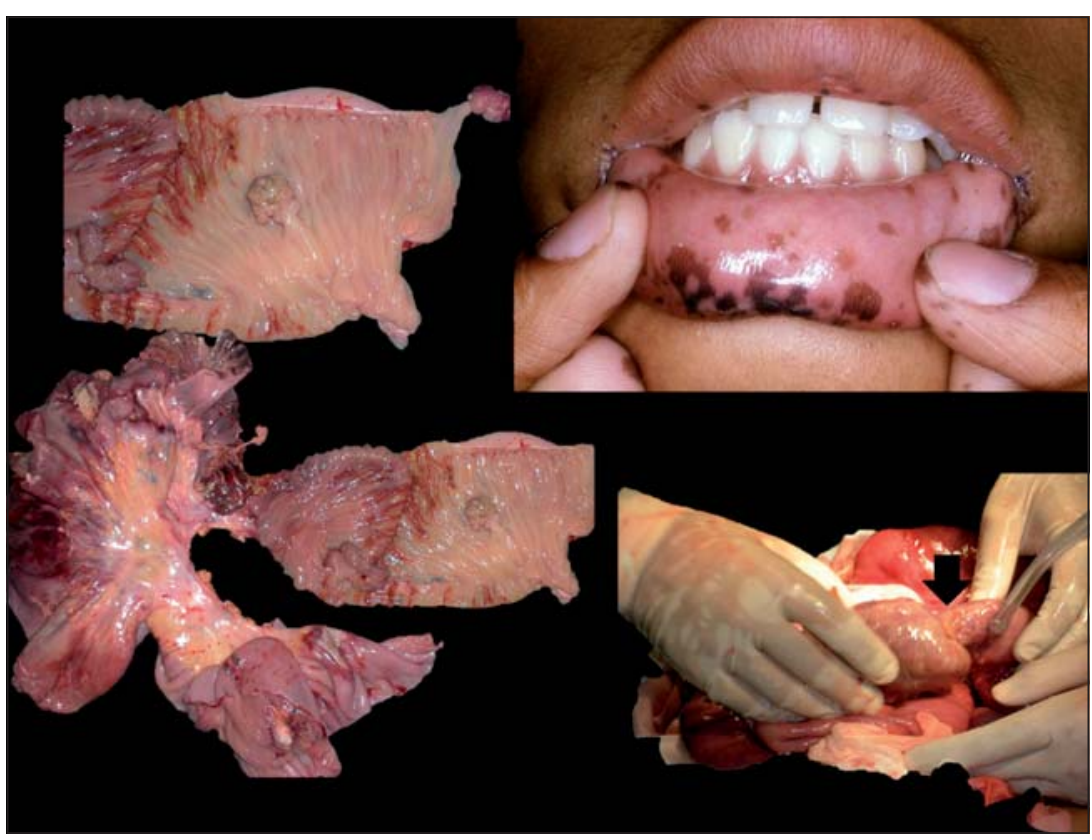

Figura 4. S. Peutz-Jeghers: invaginación y múltiples pólipos (hamartomas). positivo intenso difuso en células neoplásicas, CD3 y CD 138 negativos. Linfoma no Hodgkin difuso de células grandes $\mathrm{B}$ de alto grado.

Los casos de hamartomas corresponden a un paciente varón de 32 años y una mujer de 27 años portadores de un síndrome de Peutz-Jeghers, intervenidos previamente por invaginación intestinal a los 10 y 12 años, respectivamente. La TC demostró una masa tumoral en yeyuno proximal y fueron sometidos a una resección segmentaria de 44 y 90 $\mathrm{cm}$, respectivamente, con anastomosis inmediata y sin complicaciones (Figura 4). El estudio histopatológico reveló múltiples lesiones sésiles y pediculadas de 0,3 a $8 \mathrm{~cm}$ en un caso y de 1,5 a $3 \mathrm{~cm}$ en el otro. Ambos pacientes están en buenas condiciones luego de un seguimiento de 56 y 26 meses respecti- 
vamente, con enfermedad residual en el estómago, duodeno y colon.

Un paciente varón de 49 años consulta por cuadro de dolor abdominal cólico de 2 meses de evolución, baja de peso de 14 kilos. La TC reveló una invaginación intestinal debido a una masa tumoral de íleon distal, lo que fue confirmado en la laparotomía. Se realizó una resección segmentaria con anastomosis inmediata. La pieza de 36 cm mostró una lesión polipoidea ulcerada de $5,5 \times 5,3 \mathrm{~cm}$, pediculada constituida por células fusadas de núcleos uniformes con estroma mixoide, vasos sanguíneos sin atipia e infiltrado inflamatorio mixto. La lesión se ubica en la submucosa y compromete hasta la subserosa. IHQ: CD 117, Proteína S-100, AML y CD 34 negativos en las células fusadas. Diagnóstico: pólipo fibroide inflamatorio, ulcerado. Se mantiene asintomático a los 24 meses de intervenido.

Un paciente de 83 años con un adenocarcinoma de íleon debuta con una obstrucción intestinal y con compromiso de vejiga, falleciendo a los 24 meses por enfermedad diseminada luego de una resección segmentaria con fines paliativos. Una paciente de 72 años con un cuadro de obstrucción intestinal se interviene con el diagnóstico de masa tumoral en íleon distal confirmado por la TC abdominal. Se efectuó una resección paliativa con anastomosis inmediata por compromiso peritoneal avanzado. La biopsia revela un tumor sólido de $6 \times 4 \mathrm{~cm}$, transmural, ulcerado, con necrosis en el 30\% del volumen tumoral, con compromiso del mesenterio que corresponde a un leiomiosarcoma, con un recuento de 22 mitosis en 10 campos de aumento mayor. IHQ: CD 117 negativo; CD 34 negativo; S-100 negativo; AML positivo en el $50 \%$ de las células neoplásicas. La paciente fallece a los 4 meses.

\section{Discusión}

En esta serie el porcentaje de lesiones malignas del intestino delgado alcanza al 75\%, lo que coincide con la mayoría de las series quirúrgicas publicadas $^{9-12}$. Este porcentaje es muy inferior en estudios de autopsia, lo que confirma que la mayoría de las lesiones benignas del yeyuno-íleon son asintomáticas. Hay predominio claro del sexo masculino (20/4) y la distribución por edad es variable según el tipo de tumor (promedio 55,5 años en los GIST, 64 años en los tumores carcinoides y 50 años en los linfomas). El tumor más frecuente fue el GIST con 7 casos, de localización proximal, lo que es coincidente con otros estudios nacionales ${ }^{13,14}$ y extranjeros que excluyen los tumores de duodeno, localización donde claramente predomina el adenocarcinoma ${ }^{4,15,16}$. La hemorragia digestiva baja fue la principal mani- festación de los GIST, seguida de la masa palpable, lo que coincide con otras experiencias ${ }^{17,18}$. Esto se explica por el crecimiento exofítico de la mayoría de estas lesiones que, alcanzado cierto tamaño, se ulceran y sangran. En los tumores carcinoides, cuya lesión principal es una lesión dura tipo placa de dimensiones moderadas pero con un gran componente mesentérico, se produce una obstrucción intestinal incompleta por retracción y angulación de la pared intestinal, lo que clínicamente se traduce en un cuadro de instalación crónica con dolor, baja de peso y marcado compromiso del estado general. El adenocarcinoma y los leiomiosarcomas del yeyunoíleon, que tienen un crecimiento anular estenosante, habitualmente se diagnostican en el curso de una laparotomía exploradora por una obstrucción intestinal aguda, como ocurrió en los casos de esta serie. La escasa incidencia del adenocarcinoma en esta serie se debe a la exclusión de los tumores de duodeno, sin duda más frecuentes que los tumores distales del intestino delgado ${ }^{1,3-5,12,15,16}$. Los linfomas primarios a la macroscopia se expresan como lesiones ulceradas de gran tamaño, lo que se traduce clínicamente en la tendencia a la perforación y en menor grado la hemorragia. Los hamartomas y las lesiones polipoideas benignas tienden a producir una invaginación entero-enteral o ileocecal de instalación brusca, a veces con resolución espontánea, lo que explica la larga data de los síntomas. Al comparar las lesiones malignas $(n=18)$ con las benignas $(n=6)$ desde un punto de vista sintomático, predomina la HDB, la perforación, la anemia y la baja de peso en las primeras, siendo frecuente en ambos el dolor abdominal y la obstrucción intestinal. Sin embargo, es posible establecer un patrón clínico dependiente de la etiología del tumor, lo que se demuestra claramente en esta pequeña serie.

Con la aparición de la TC y, especialmente de la enteroclisis por TC, el diagnóstico preoperatorio de tumor de ID en la actualidad es algo habitual, como lo demuestra esta serie. La TC, efectuada en 20 pacientes reveló una masa o engrosamiento parietal en todos ellos, además del compromiso del mesenterio y de otros órganos. Los GIST se manifiestan como una masa sólida exofítica y heterogénea con intenso realce, aunque en lesiones de gran tamaño, puede expresarse como una masa sólido-quística por la necrosis central del tumor, como ocurrió en uno de nuestros casos. Es característico en los tumores carcinoides avanzados el aspecto radiado que adquieren los vasos del mesenterio, secundario a la reacción desmoplástica que determina la fibrosis, retracción y angulación con dilatación variable de los segmentos proximales del intestino. Aunque puede presentar patrones muy variables, lo más llamativo en el linfoma primario de ID es el engrosamiento parietal cir- 
cunferencial con infiltración del mesenterio (Figura 5). Por ello, el compromiso intestinal de un linfoma retroperitoneal es difícil diferenciar de un tumor primario. La imagen característica del adenocarcinoma es un engrosamiento focal de la pared intestinal que provoca una estenosis con grados variables de obstrucción proximal ${ }^{19}$. Las ventajas de la enteroclisis por TC es su capacidad para mostrar la masa tumoral que afecta el intestino y/o el mesenterio, es decir, mostrar en forma simultánea alteraciones intraluminales, parietales y extraintestinales, así como compromiso de otros órganos a distancia y es especialmente útil en los pacientes con obstrucción intestinal ${ }^{2,19}$.

Considerado en forma global, la localización más frecuente fue el íleon (14 lesiones), sin diferencias entre lesiones malignas y benignas, similar a otras series nacionales ${ }^{3,13,14}$. Es llamativo el compromi-
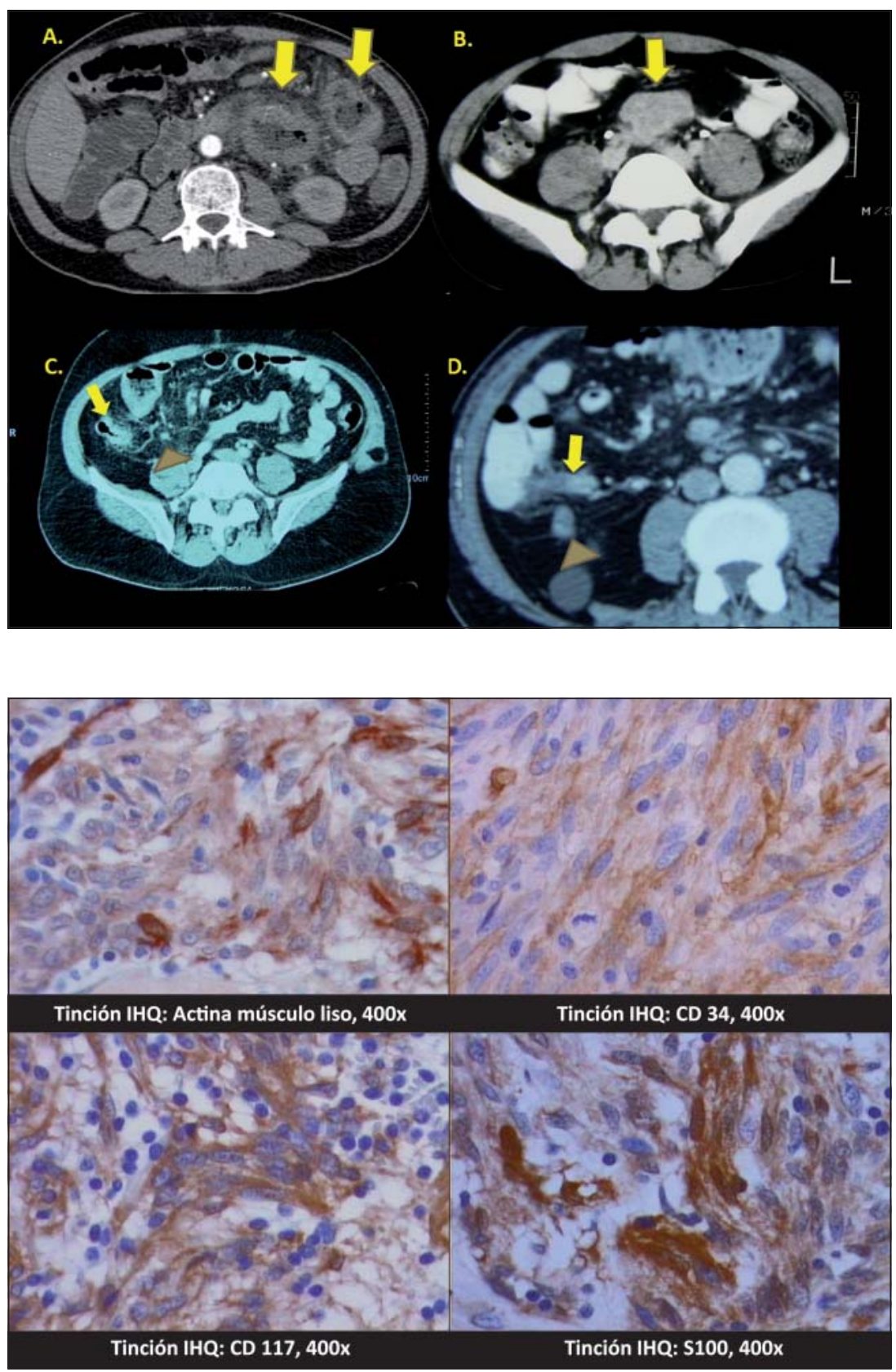

Figura 5. A. Enteroclisis por TC: engrosamiento marcado del yeyuno proximal. B. GIST: masa sólida. C y D. Carcinoide del íleon: engrosamiento pared del intestino con gran reacción desmoplástica del mesenterio.
Figura 6. IHQ de tumor GIST: tinción positiva focal en células neoplásicas para AML, CD 34 y S 100; marcadamente positiva para CD 117. 
so del yeyuno y de íleon proximal de los GIST mientras que los tumores carcinoides y el linfoma afectan de preferencia al íleon terminal. Alrededor del 30 a $40 \%$ de los tumores carcinoides del ID son múltiples, lo que ocurrió en 4 de los 6 pacientes en nuestra serie, multiplicidad que también es frecuente en los linfomas.

La cirugía es el pilar del tratamiento de los tumores primarios del ID, idealmente mediante resecciones radicales en block, lo que no siempre es factible. Los GIST debido al tipo de crecimiento exofítico sólo requieren resecciones segmentarias sin gran linfadenectomía, lo que es básico en los adenocarcinomas y en los carcinoides que tienen un gran componente tumoral mesentérico. El compromiso linfonodal es relevante en los adenocarcinomas y los pacientes resecados sin ganglios afectados tienen una sobrevida a 5 años de 70 a 80\%. En los GIST los criterios de malignidad incluyen el tamaño de la lesión, en número de mitosis por campo y el grado de necrosis tumoral. El carcinoide tiene un comportamiento biológico muy variable y en 30 a $40 \%$ de los casos son lesiones múltiples, como sucede en esta serie $^{20}$. Los factores pronósticos más destacados de los tumores carcinoides son las metástasis a distancia, el índice mitótico, la multiplicidad, el síndrome carcinoide y la profundidad de la lesión ${ }^{4,15,20}$. En nuestra experiencia, los linfomas primarios de ID se presentan como emergencias quirúrgicas en etapas muy avanzadas con un pronóstico ominoso.

El pronóstico de estas lesiones depende del tipo histológico, el grado de malignidad y la etapa al momento del diagnóstico. En esta serie, el carcinoide debuta con una obstrucción intestinal y la cirugía radical fue curativa. En los tumores GIST dos pacientes presentan una recidiva a distancia (uno de ellos con recidiva loco-regional) y fallecen por la enfermedad. Los 5 casos de linfomas debutan con una perforación en tres casos, una HDBM y una obstrucción intestinal alta, el diagnóstico se sospechó por el TC en 3 y la laparotomía en 2, 4 afectaron al íleon y 1 al yeyuno proximal, 3 tenían lesiones múltiples destacando la ulceración y engrosamiento parietal marcado y el diagnóstico fue confirmado por la IHQ. Todos los pacientes tenían una enfermedad localmente avanzada y/o diseminada, lo que explica el ominoso pronóstico de estos tumores, lo que es concordante con otras series ${ }^{22}$.

La mayoría de los estudios analizan los síntomas de los tumores de intestino delgado en forma global (incluidos los tumores de duodeno) o bien categorizados por la malignidad ${ }^{3}$. A pesar de la escasa frecuencia de estas lesiones, es posible reconocer cierta correlación entre el tipo histológico, la localización y la forma clínica de presentación de los tumores primarios de yeyuno-íleon. Aunque la heterogeneidad es amplia, podemos concluir que predomina la HDB en los GIST, la obstrucción intestinal incompleta en los carcinoides, la perforación en los linfomas, la obstrucción aguda en los adenocarcinomas y la intususcepción en las lesiones benignas $^{11,21-24}$. La enteroclisis por TC es actualmente el examen preoperatorio más relevante ${ }^{2,19}$. El GIST se desarrolla preferentemente en segmentos proximales de yeyuno, mientras que el tumor carcinoide y el linfoma afectan principalmente al íleon distal ${ }^{11}$. Excluyendo el duodeno, el GIST y los tumores carcinoides representan más de la mitad del global y el 65\% de las neoplasias malignas primarias que afectan este segmento del tubo digestivo. El estudio IHQ es relevante en el diagnóstico de certeza de este tipo de tumores (Figura 6). El 75\% de los tumores de yeyuno e íleon son malignos y el pronóstico es muy variable, dependiendo del tipo histológico y del estadio de la enfermedad al momento de la cirugía ${ }^{25}$.

\section{Referencias}

1. Disario JA, Burt RW, Vargas H, McWhorter WP. Small bowel cancer: epidemiological and clinical characteristics from a population-based registry. Am J Gastroenterol. 1994;89:699-701.

2. Besa C, O'Brien A, Cruz JP. Utilidad de la enteroclisis por tomografía computada en la detección de tumores de intestino delgado. Rev Chil Radiol. 2008;14:212-8.

3. Valera JM, Maiza E, Contreras L, Smok G. Tumores de intestino delgado: experiencia de 11 años en un centro de referencia. Gastr Latinoam. 2004;15:180-5.

4. Hatzaras I, Palesty A, Abir F, Sullivan P, Kozol RA, Dudrick SJ, et al. Small-bowel tumors. Epidemiologic and clinical characteristics of 1260 cases. Arch Surg. 2007;142:229-35.

5. Han S-L, Cheng J, Zhou H-Z, Guo S-C, Jia Z-R, Wang P-F. Surgically treated primary malignant tumor of small bowel: a clinical analysis. World J Gastroenterol. 2010;16:1527-32.

6. AJCC Cancer Staging Manual. $6^{\text {th }}$ edition. New York, Springer, 2002:107-10.

7. Harris NL, Jaffe ES, Diebold J, Flandrin G, MullerHermelink HK, Vardiman J, et al. World Health Organization classification of neoplastic diseases of the hematopoietic and lymphoid tissues: report of the Clinical Advisory Committee meeting-Airlie House, Virginia. J Clin Oncol. 1999;17:3835-49.

8. Fletcher CD, Berman JJ, Corless C, Gorstein F, Lasota J, Longley BJ. Diagnosis of gastrointestinal stromal tumors: a consensus approach. Int J Surg Pathol. 2002;10:81-9.

9. Yang Y-S, Huang Q-Y, Wang P-F, Sun G, Peng L-H. Primary jejunoileal neoplasms: a review of 60 cases. World J Gastroenterol. 2003;9:862-4. 
10. Yang WL, Zhang XC, Yan ZQ, Zhang HM, Zhao Z, Zhang JG, et al. Clinical analysis of primary small intestinal neoplasms in 305 cases. Zhonghua Zhong Liu Za Zhi 2007;29:781-3.

11. Kim CHK, Kye BH, Lee JI, Kim SH, Kim HJ, Kang WK, et al. Clinicopathological features of primary jejunoileal tumors. J Korean Soc Coloproctol. 2010;26:3348.

12. Egberts JH, Scharrer ML, Hinz S, Schafmayer C, Klomp HJ, Faendrich F, et al. Small bowel cancer: single-centre results over a period of 12 years. Hepatogastroenterology 2007;54:129-34.

13. Beltrán M, Cruces KS. Primary tumors of jejunum and ileum as a cause of intestinal obstruction: a case control study. Int J Surg. 2001;5:183-91.

14. Rojas J, Pereira N, Cortés S, Ferreira A, Pereda L, Sfeir $\mathrm{P}$, y cols. Tumores de intestino delgado: epidemiología y características clínicas en un hospital universitario. Libro Resúmenes Congreso Chileno de Cirugía 2010:71.

15. Chow JS, Chen CC, Ahsan H, Neugut AI. A population-based study of the incidence of malignant small bowel tumours: SEER, 1973-1990. Int J Epidemiology 1996;25:722-7.

16. Stang A, Stegmaier C, Eisinger B, Stabenow R, Metz KA, Jöckel K-H. Descriptive epidemiology of small intestinal malignancies: the German Cancer Registry experience. Br J Surg. 1999;80:1440-4.

17. Lakshmi VA, Chacko RT, Kurian S. Gastrointestinal stromal tumors: a 7-year experience from a tertiary care hospital. Indian J Pathol Microbiol. 2010;53:628-33.

18. Fernández Salazar LI, Alvarez Gago T, Sanz Rubiales A, Velayos Jiménez B, Aller de la Fuente R, González Hernández JM. Tumores del estroma gastrointestinal (GIST): aspectos clínicos.

19. Sailer J, Zacherl J, Schima W. MDCT of small bowel tumours. Cancer Imaging 2007;7:224-33.

20. Burke AP, Thomas RM, Elsayed AM, Sobin LH. Carcinoids of the jejunum and ileum. Cancer 1997;79:108393.

21. Blanchard DK, Budde JM, Hatch GF, Wertheimer-Hatch L, Hatch KF, Davis GB, et al. Tumors of the small intestine. World J Surg. 2000;24:421-9.

22. Ara C, Coban S, Kayaalp C Yilmaz S, Kirimlioglu V. Spontaneous intestinal perforation due to nonHodgkin's lymphoma: evaluation of 8 cases. Dig Dis Sci. 2007;52:1752-6.

23. Ramia JM, Sancho E, Lozano O, Santos JM, Garrido F. Linfoma primario de intestino delgado. Cir Esp. 2007;81:46-8.

24. Blanchard DK, Budde JM, Hatch GF 3rd, WertheimerHatch L, Hatch KF, Davis GB, et al. Tumors of the small intestine. World J Surg. 2000;24:421-9.

25. Farhat MH, Shamseddine AI, Barada KA. Small bowel tumors: clinical presentation, prognosis, and outcome in 33 patients in a tertiary care center. J Oncol. 2008; doi:10.1155/2008/212067. 